\title{
NONLINEAR FUNCTIONAL EQUATIONS IN BANACH SPACES AND HOMOTOPY ARGUMENTS ${ }^{1}$
}

\author{
BY PETER HESS
}

Communicated by Felix Browder, July 13, 1970

Let $T$ be a (nonlinear) mapping from the real reflexive Banach space $X$ into its conjugate space $X^{*}$. We investigate the solvability of the functional equation $T u=0$ in a given open bounded subset $\Omega$ of $X$. If the mapping $T$ is coercive, existence theorems have been derived for $T$ belonging to various classes of nonlinear mappings of monotone type. We consider here the situation where the mapping $T=A_{0}$ is (in an appropriate sense) homotopic to an odd operator $A_{1}$. Taking the whole set $\Lambda$ of finite-dimensional subspaces of $X$ rather than an injective approximation scheme, we do not assume the separability of the space $X$. We further work with only mild continuity assumptions on the mappings involved and do not suppose their boundedness. Using an approximation of a maximal monotone mapping by single-valued, everywhere defined maximal monotone operators due to Brézis-Crandall-Pazy [3], we are able to derive results based on homotopy arguments also for multi-valued, not everywhere defined mappings. We then apply our results to asymptotically homogeneous mappings and obtain some kind of Fredholm alternative for the existence of solutions of the equation $T u=f$. A Fredholm alternative for nonlinear equations of Hammerstein type is finally stated. Our theorems extend results by Browder [5], [6], [7], Browder-Petryshyn [10], Figueiredo-Gupta [11], Nečas [15] and others. We note that we assume for the homotopy $A(u, t): X$ $\times[0,1] \rightarrow X^{*}$ that it is continuous in $t$, uniformly with respect to $u$ in bounded sets. A homotopy argument which weakens this assumption (but demands the continuity of the mappings) is given by the writer in [13].

Before stating our main results, we put together some notations and definitions we employ. If $X$ is a real Banach space, $X^{*}$ its conjugate space, $(v, u)$ denotes the duality pairing between $v \in X^{*}$

AMS 1969 subject classifications. Primary 3930, 4780; Secondary 4610, 5540.

Key words and phrases. Nonlinear functional equation, reflexive Banach space, homotopy arguments, odd operators, unbounded mappings, maximal monotone mappings, asymptotically homogeneous operators, Fredholm alternative, Hammerstein equation.

1 This research was partially supported by N.S.F. Grant GP-23563. 
and $u \in X$. A subset $G$ of the product space $X \times X^{*}$ is monotone, if $\left(v_{1}-v_{2}, u_{1}-u_{2}\right) \geqq 0$ for all $\left[u_{1}, v_{1}\right] \in G,\left[u_{2}, v_{2}\right] \in G$. A monotone set is said to be maximal monotone, if it is not properly contained in any other monotone set. For a mapping $T$ of $X$ into the set $2^{X^{*}}$ of all subsets of $X^{*}$, the effective domain $D(T)$ is given by $D(T)$ $=\{u \in X: T u \neq \varnothing\}$. The graph $G(T)$ of $T$ is then the subset of $X \times X^{*}$ given by $G(T)=\left\{[u, v] \in X \times X^{*}: u \in D(T), v \in T u\right\} . T$ is said to be monotone if its graph $G(T)$ is a monotone set, and maximal monotone if $G(T)$ is maximal monotone. We say that $T: X \rightarrow 2^{X^{*}}$ is odd if $[-u,-v] \in G(T)$ provided $[u, v] \in G(T)$, and $\mathfrak{x}$-homogeneous $(\mathfrak{x}>0)$, if $[u, v] \in G(T)$ implies that $\left[t u, t^{ \pm} v\right] \in G(T)$ for $t>0$. Further a (singlevalued) mapping $T$ of $X$ into $X^{*}$ is demicontinuous, if it is continuous from the strong topology on $X$ to the weak topology on $X^{*}$. An operator $T: X \rightarrow X^{*}$ has property $(\mathrm{S})^{+}$if each sequence $\left\{u_{n}\right\} \subset X$ converging weakly to some element $u$ such that $\lim \sup \left(T u_{n}, u_{n}-u\right) \leqq 0$ possesses a subsequence $\left\{u_{n_{k}}\right\}$ converging strongly to $u$. Mappings with property $(S)^{+}$have been introduced by Browder [4] in the study of nonlinear eigenvalue problems, and have been of great importance in any kind of homotopy consideration since. We finally say that a mapping $T: X \rightarrow 2^{X^{*}}$ is quasi-bounded [9] if for each $M>0$ there exists $K(M)>0$ such that $\|u\| \leqq M$ and $(v, u) \leqq M\|u\|$ for $v \in T u$ imply that $\|v\| \leqq K(M)$. It is proved in [9] that for example monotone operators having 0 as an interior point of their domain are quasi-bounded.

TheOREM 1. Let $X$ be a real reflexive Banach space and $\Omega$ an open bounded subset of $X$ containing 0 and symmetric about the origin. Let $A_{t} u=A(u, t)$ be a mapping of $X \times[0,1]$ into $X^{*}$ having the following properties:

(i) for each fixed $t, A_{t}$ is a demicontinuous mapping with property (S)+;

(ii) $A_{t} u$ is continuous in $t$, uniformly for $u$ in the closure $\operatorname{cl}(\Omega)$ of $\Omega$ in $X$;

(iii) $A_{1}$ is odd.

Suppose that $A_{t} u \neq 0$ for all $u$ in the boundary bdry $(\Omega)$ of $\Omega$ and all $t \in[0,1]$. Then the equation $A_{0} u=0$ has a solution $u_{0}$ in $\Omega$.

Theorem 1 follows by the classical Borsuk-Ulam theorem [2], [14], the invariance of the Brouwer degree under suitable homotopies, and arguments which have become standard in the theory of mappings of monotone type from

LEMma 1. Under the assumptions of Theorem 1, there exists a $F \in \Lambda$ such that for all $F^{\prime} \in \Lambda$ with $F^{\prime} \supset F$, all $u_{F^{\prime}} \in \operatorname{bdry}(\Omega) \cap F^{\prime}$ and all $t \in[0,1], A_{t, F^{\prime}} u_{F^{\prime}} \neq 0$. 
Here we denote by $A_{t, F}: F \rightarrow F^{*}$ the approximant of $A_{t}$ defined by $A_{t, F}=j_{F}^{*} A_{t} j_{F}$, where $j_{F}$ is the injection mapping of $F$ into $X$. Under the additional restriction that the mappings $A_{t}$ are bounded (i.e. map bounded sets onto bounded sets), Lemma 1 has been obtained by Nečas [15]. We briefly sketch the

Proof of Lemma 1. Because of the uniform continuity of $A_{t} u$ in $t$ with respect to $u \in \operatorname{cl}(\Omega)$, it suffices to show that for each fixed $t_{0} \in[0,1]$ there exist $\epsilon>0$ and $F \in \Lambda$ such that $\left\|A_{t_{0}, F^{\prime}} u_{F^{\prime}}\right\| \geqq \epsilon$ for all $F^{\prime} \in \Lambda, F^{\prime} \supset F$, and all $u_{F^{\prime}} \in \operatorname{bdry}(\Omega) \cap F^{\prime}$. We assume, on the contrary, that the set

$$
V_{\epsilon, F}=\left\{u_{F^{\prime}}: F^{\prime} \in \Lambda, F^{\prime} \supset F, u_{F^{\prime}} \in \operatorname{bdry}(\Omega) \cap F^{\prime},\left\|A_{t_{0}, F^{\prime}} u_{F^{\prime}}\right\|<\epsilon\right\}
$$

is nonempty for each $\epsilon>0$ and each $F \in \Lambda$. For fixed $F \in \Lambda$, the family $\left\{V_{1 / n, F}\right\}_{n=1}^{\infty}$ has the finite intersection property. Therefore, by the boundedness of $\Omega$ and the reflexivity of the space $X, W_{F}$ $=\bigcap_{n=1}^{\infty}\left\{\right.$ weak cl $\left.V_{1 / n, F}\right\}$ is nonempty. The family $\left\{W_{F}\right\}_{F \in \Delta}$ has again the property of finite intersections; consequently there exists $u_{0} \in \bigcap_{F \in \Lambda} W_{F}$. For an arbitrary element $w \in X$, we choose $F_{0} \supset\left\{u_{0}, w\right\}$. By a diagonalization process, we can find a sequence $\left\{u_{n}\right\}$ converging weakly to $u_{0}$, with $u_{n} \in \operatorname{bdry}(\Omega) \cap F_{n}, F_{n} \in \Lambda, F_{n} \supset F_{0}$, such that $\left\|A_{t_{0}, F_{n}} u_{n}\right\|<1 / n$. Hence $\lim _{n \rightarrow \infty}\left(A_{t_{0}} u_{n}, u_{n}-u_{0}\right)=0$, and property (S) ${ }^{+}$of the mapping $A_{t_{0}}$ guarantees the existence of a subsequence of $\left\{u_{n}\right\}$ converging strongly to $u_{0}$. We infer that $u_{0} \in \operatorname{bdry}(\Omega)$, and that $\left(A_{t_{0}} u_{0}, u_{0}-w\right)=0$. This last relation being true for all $w \in X$, we obtain $A_{t_{0}} u_{0}=0$, in contradiction to the assumptions of Theorem 1. Q.E.D.

We now pass to existence theorems for mappings of the form $T+A$, where $T: X \rightarrow 2^{X^{*}}$ is maximal monotone, and where $A$ satisfies condition $(\mathrm{S})^{+}$. Our main result is

TheOREM 2. Let $X$ be a real reflexive Banach space, $\Omega$ an open bounded subset of $X$ containing 0 and symmetric about 0 . Let $T: X \rightarrow 2^{X^{*}}$ denote an odd maximal monotone mapping with $0 \in D(T)$, and let finally $A_{t} u=A(u, t): X \times[0,1] \rightarrow X^{*}$ be a mapping with the following properties:

(i) for each fixed $t, A_{t}$ is a demicontinuous, quasi-bounded mapping satisfying condition $(\mathrm{S})^{+}$;

(ii) $A_{t} u$ is continuous in $t$, uniformly for $u \in \operatorname{cl}(\Omega)$;

(iii) $A_{1}$ is odd.

Let $0 \in\left(T+A_{t}\right) u$ for all $u \in \operatorname{bdry}(\Omega) \cap D(T)$ and all $t \in[0,1]$. Then there exists $u_{0} \in \Omega$ with $0 \in\left(T+A_{0}\right) u_{0}$.

By a result of Asplund [1] we may assume without loss of gen- 
erality that $X$ and $X^{*}$ are strictly convex. The idea of proof of Theorem 2 is to reduce it to Theorem 1 by approximating $T$ by odd bounded maximal monotone mappings $T_{\lambda}(\lambda>0)$ which, under our assumptions on $X$ and $X^{*}$, are single-valued, and which are defined on the whole of $X$ as follows:

$T_{\lambda} u=\left\{v \notin X^{*}:\right.$ there exists $x \in D(T)$ such that $\left.v \in T x, \lambda v=J(u-x)\right\}$.

Here $J$ denotes the duality-mapping of $X$ into $X^{*}$ :

$$
J u=\left\{q \in \alpha^{*}:(q, u)=\|q\|\|u\|,\|q\|=\|u\|\right\} .
$$

This approximation method is due to Brézis-Crandall-Pazy [3] and is improved and extended by Browder and the writer in [9]. The reduction to Theorem 1 is guaranteed by

LeMma 2. The hypotheses of Theorem 2 imply the existence of a constant $\lambda_{0}>0$ such that $\left(T_{\lambda}+A_{t}\right) u \neq 0$ for all $u \in \operatorname{bdry}(\Omega), t \in[0,1]$ and $\lambda \in\left(0, \lambda_{0}\right)$.

The proof of Lemma 2 is based on results on generalized pseudomonotone operators derived in [9]. We note that, for each fixed $\lambda \in\left(0, \lambda_{0}\right)$, the mapping $T_{\lambda}+A_{t}$ then satisfies the assumptions of Theorem 1. Hence there exists $u_{\lambda} \in \Omega$ with $0=\left(T_{\lambda}+A_{0}\right) u_{\lambda}$. By a limiting process $\lambda \downarrow 0$, we obtain a solution of the given equation.

We now apply Theorem 2 in order to get results of Fredholm alternative type for asymptotically homogeneous operators. The following theorem allows interesting applications to boundary value problems for nonlinear elliptic equations with degenerate coefficients (for the linear case see $[12])$ :

TheOREM 3. Let $T$ be an odd, $\mathfrak{x}$-homogeneous maximal monotone mapping from the real reflexive space $X$ into $2^{X^{*}}$. Let further $A: X \rightarrow X^{*}$ be a quasi-bounded, demicontinuous, odd and $\mathfrak{x}$-homogeneous mapping with property $(\mathrm{S})^{+}$, and let $N: X \rightarrow X^{*}$ be bounded, demicontinuous, with $\lim \|u\|_{\rightarrow \infty}\|N u\| /\|u\|^{\mathfrak{r}}=0$, and such that $A+N$ has property $(\mathrm{S})^{+}$. If $0 \in(T+A) u$ implies that $u=0$, then $T+A+N$ has range $X^{*}$.

This theorem extends a corresponding result by Nečas [15]. Another application of Theorem 2, together with arguments derived in [8], gives the following Fredholm alternative for Hammerstein equations:

TheOREM 4. Let the Banach space $X$ be real and reflexive, $T: X^{*} \rightarrow 2^{X}$ maximal monotone, odd and (1/x)-homogeneous (x $>0), A: X \rightarrow X^{*}$ odd, $\mathfrak{r}$-homogeneous with property $(S)^{+}$, and $N: X \rightarrow X^{*}$ bounded, demi- 
continuous with $\lim \left\|_{u}\right\|_{\rightarrow \infty}\|N u\| /\|u\|^{\mathfrak{x}}=0$, and such that $A+N$ has property (S)+. Assume in addition that $A$ is uniformly continuous on bounded sets (and hence bounded). If $0 \in u+T A u$ only for $u=0$, then the equation $f \in u+T(A+N) u$ admits a solution for all $f \in X$.

\section{REFERENCES}

1. E. Asplund, Averaged norms, Israel J. Math. 5 (1967), 227-233. MR 36 \#5660.

2. K. Borsuk, Drei Sätze über die n-dimensionale Euklidische Sphäre, Fund. Math. 20 (1933), 177-190.

3. H. Brézis, M. Crandall and A. Pazy, Perturbations of nonlinear maximal monotone sets in Banach space, Comm. Pure Appl. Math. 23 (1970), 123-144.

4. F. E. Browder, Nonlinear eigenvalue problems and Galerkin approximations, Bull. Amer. Math. Soc. 74 (1968), 651-656. MR 37 \#2043.

5. - Nonlinear operators and nonlinear equations of evolution in Banach spaces, Proc. Sympos. Pure Math., vol. 18, part 2, Amer. Math. Soc., Providence, R.I. (to appear).

6. - Existence theorems for nonlinear partial differential equations, Proc. Sympos. Pure Math., vol. 16, Amer. Math. Soc., Providence, R.I., 1970.

7. - Nonlinear elliptic boundary value problems and the generalized topological degree, Bull. Amer. Math. Soc. 76 (1970), 999-1005.

8. F. E. Browder, D. G. de Figueiredo and C. P. Gupta, Maximal monotone operators and nonlinear integral equations of Hammerstein type, Bull. Amer. Math. Soc. 76 (1970), 700-705.

9. F. E. Browder and P. Hess, Nonlinear mappings of monotone type in Banach spaces (to appear).

10. F. E. Browder and W. V. Petryshyn, Approximation methods and the generalized topological degree for nonlinear mappings in Banach spaces, J. Functional Analysis 3 (1969), 217-245. MR 39 \#6126.

11. D. G. de Figueiredo and C. P. Gupta, Borsuk type theorems for nonlinear noncompact mappings in Banach spaces (to appear).

12. P. Hess, Ueber das verallgemeinerte Dirichletproblem für lineare partielle Differentialgleichungen, Ann. Acad. Sci. Fenn. AI, No. 434 (1969).

13. - Nonlinear operator equations and eigenvalue problems in nonseparable Banach spaces (to appear).

14. M. A. Krasnosel'skir, Topological methods in the theory of nonlinear integral equations, GITTL, Moscow, 1956; English transl., Macmillan, New York, 1964. MR 20 \#3464; MR 28 \#2414.

15. J. Nečas, $A$ remark on the Fredholm alternative for nonlinear operators with application to nonlinear integral equations of generalized Hammerstein type (to appear).

University of Chicago, Chicago, Illinois 60637 